\title{
Superoxide Dismutase 1 Regulation of CXCR4-Mediated Signaling in Prostate Cancer Cells is Dependent on Cellular Oxidative State
}

\author{
Brent Young Chad Purcell Yi-Qun Kuang Nicholle Charette Denis J. Dupré \\ Department of Pharmacology, Faculty of Medicine, Dalhousie University, Halifax, NS, Canada
}

\section{Key Words}

Superoxide dismutase $1 \cdot$ CXCR4 - Prostate cancer $\cdot$ Signaling $\cdot$ G protein-coupled receptor $\bullet$ Cell migration

\begin{abstract}
Background/Aims: CXCL12, acting via one of its G protein-coupled receptors, CXCR4, is a chemoattractant for a broad range of cell types, including several types of cancer cells. Elevated expression of CXCR4, and its ligand CXCL12, play important roles in promoting cancer metastasis. Cancer cells have the potential for rapid and unlimited growth in an area that may have restricted blood supply, as oxidative stress is a common feature of solid tumors. Recent studies have reported that enhanced expression of cytosolic superoxide dismutase (SOD1), a critical enzyme responsible for regulation of superoxide radicals, may increase the aggressive and invasive potential of malignant cells in some cancers. Methods: We used a variety of biochemical approaches and a prostate cancer cell line to study the effects of SOD1 on CXCR4 signaling. Results: Here, we report a direct interaction between SOD1 and CXCR4. We showed that SOD1 interacts directly with the first intracellular loop (ICL1) of CXCR4 and that the CXCL12/ CXCR4-mediated regulation of AKT activation, apoptosis and cell migration in prostate cancer (PCa) cells is differentially modulated under normal versus hypoxic conditions when SOD1 is present. Conclusions: This study highlights a potential new regulatory mechanism by which a sensor of the oxidative environment could directly regulate signal transduction of a receptor involved in cancer cell survival and migration.
\end{abstract}




\section{Cellular Physiology Cell Physiol Biochem 2015;37:2071-2084

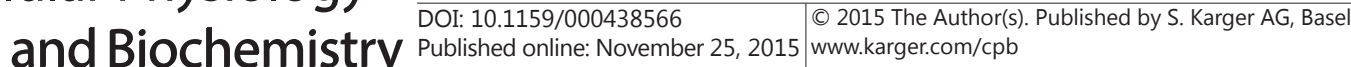 \\ Young et al.: SOD1 Regulation of CXCR4 Signaling}

\section{Introduction}

Expression levels of CXCL12 and its receptor CXCR4 are higher in human prostate cancer (PCa) tissue than those in normal or benign prostatic hyperplasia tissues [1]. Patients with a high expression of CXCR4 in tumours have shorter survival times and more aggressive cancer phenotypes than those with lower CXCR4 levels [2]. Interaction of CXCL12 with CXCR4 plays a role in the metastasis of PCa to both bone $[3,4]$ and neural tissue $(1)$, as well as the metastasis of other cancer types including neuroblastoma, rhabdomyosarcoma, and breast and ovarian cancers [5-10]. CXCL12 exhibits peak levels of expression in organs that are common destinations of cancer metastasis, including the bone marrow (6). While CXCR4 expression is elevated in cancer cells, its expression is minimal in normal adjacent tissues, if it is expressed at all $[4,6]$. The changes in receptor expression levels have been suggested to result from changes in the adjacent vasculature that bring hypoxic conditions during tumour progression [11]. Hypoxia is known to promote metastasis and increases CXCR4 expression during cancer progression $[12,13]$. Due to the involvement of the CXCR4/CXCL12 axis in cell migration, angiogenesis, and development, this signaling pathway is often exploited by cancer cells for metastasis, survival and proliferation [14]. CXCL12 promotes cell survival through phosphoinositide 3-kinase (PI3K) and MAPK cascades, even in the absence of cellcycle progression. PI3K can lead to the activation of the serine-threonine kinase AKT, which plays a key role in tumour cell survival and possibly proliferation [15]. Both p38 and ERK1/2 MAPK are also involved in tumour cell survival [16]. The pro-apoptotic Bcl-2-associated death promoter (BAD) protein can be inactivated by CXCL12-mediated activation of ERK and PI3K pathways.

Eukaryotic cells produce reactive oxygen species (ROS) as a by-product of normal metabolism. The levels of ROS (superoxide, hydrogen peroxide, and hydroxyl radicals) fluctuate in cells and can accumulate during increased metabolism or upon exposure to UV light, ionizing radiation, or chemotherapeutic agents, which in turn may lead to protein, lipid, and DNA damage. The first line of defense against ROS-induced damage is the conversion of superoxide to hydrogen peroxide by the antioxidant enzyme superoxide dismutase (SOD) [17]. Not surprisingly, disturbances in the function of SOD isoforms lead to numerous pathological changes in humans, including tumours [18]. Because tumour cells are exposed to particularly high levels of oxidative stress, SOD is expected to have an augmented impact in these cells [19]. Orally available SOD1 inhibitors were found to dampen both the inflammation-mediated effects on tumour progression and metastasis of fibrosarcoma cells, and the tumour growth and proliferation of PCa cells, demonstrating that SOD1 plays an important role in cancer progression $[20,21]$.

We identified an interaction between CXCR4 and SOD1. However, the relationship between CXCR4 signaling and SOD1 activity in controlling metastasis has not yet been characterized. Given the important role of both SOD1 and CXCR4 in metastasis, the importance of negatively regulating the activity of both SOD1 and CXCR4 under normal growth conditions, and our novel finding of a physical interaction between SOD1 and CXCR4, we hypothesize that SOD1 associates with CXCR4 to regulate CXCR4 signaling towards cell survival, apoptosis or cell migration. This interaction would regulate CXCR4 chemotactic signaling and apoptosis. However, under the hypoxic conditions found within the tumour microenvironment, SOD1 regulation of CXCR4 could change, possibly resulting in strong activation of chemotactic pathways promoting cell migration and metastasis following a CXCL12 gradient.

\section{Materials and Methods}




\section{Cellular Physiology Cell Physiol Biochem 2015;37:2071-2084

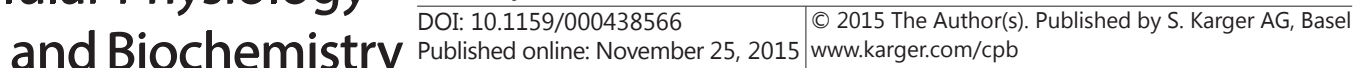 \\ Young et al.: SOD1 Regulation of CXCR4 Signaling}

from Invitrogen (Etobicoke, ON, Canada). Complete, EDTA-free Protease Inhibitor Cocktail Tablets and X-tremeGENE HP DNA Transfection Reagent (X-tremeGENE) were acquired from Roche Applied Science (Laval, QC, Canada). Dulbecco's Modified Eagle's Medium (DMEM) High Glucose, Protein A-Sepharose, and all other chemicals, unless otherwise noted, were obtained from Sigma-Aldrich (Oakville, ON, Canada). Supersignal West Femto Maximum Sensitivity Substrate was from Pierce Biotechnology (Rockford, IL, USA). Anti-SOD1, anti-fusin (C-20; anti-CXCR4), anti-phospho-FAK, anti- $\beta$-actin, anti-phospho-Akt (Ser-473), anti-c-myc, anti-HA, anti-HIF1 $\alpha$, all horseradish peroxidase (HRP)-conjugated secondary antibodies and the Annexin V Apoptosis Assay Kit were purchased from Santa Cruz Biotechnologies, Inc. (Santa Cruz, CA, USA). Bovine Serum Albumin (BSA) was purchased from BioBasic Inc. (Markham, ON, Canada). SOD1 shortinterfering ribonucleic acid (siRNA), $\beta$-Actin primary antibody and CXCL12 (SDF1 $\alpha$ ) were purchased from Abcam (Toronto, ON, Canada). Recombinant SOD1 was obtained from Creative Biomart (Shirley, NY) and G protein siRNAs and controls were purchased from Qiagen Inc. Pertussis toxin (PTX) was purchased from List Biological Laboratories, Inc. (Campbell, CA, USA).

\section{Molecular Constructs}

The pcDNA3.1 HA-CXCR4 receptor was purchased from Missouri University of Science and Technology cDNA resource center (Rolla, MO, USA). pCMV6-myc-DDK-hSOD1 wild-type (WT) was purchased from Origene Technologies Inc. (Rockville, MD, USA). CXCR4-YFP was a gift from Dr. Nikolaus Heveker, from Hôpital Ste-Justine (Montréal, QC, Canada). The $\mathrm{G}_{\mathrm{i}^{-}}-\mathrm{G} \alpha_{\mathrm{q}}-$ and $\mathrm{G} \alpha_{13}-R$ lucII constructs were obtained from Dr. Michel Bouvier, from Université de Montréal (Montréal, QC, Canada).

\section{Cell culture and Transfection}

PC3 cells were cultured in 6-well plates using DMEM supplemented with 10\% FBS and 1\% PenicillinStreptomycin under $5 \% \mathrm{CO}_{2}$ conditions. For hypoxic cell treatment, the cells were incubated in a hypoxia chamber containing $0.2 \% \mathrm{O}_{2}$ at $37^{\circ} \mathrm{C}$ for the indicated length of time (Fig. 1). The chamber was purged prior to usage to ensure that the chamber maintained the correct oxygen concentrations.

Transfections were carried out using XtremeGENE according to the instructions provided by the manufacturer (Roche). Transfection solutions were replaced with fresh FBS-free media 24 hours posttransfection. Transient transfection of SOD1 siRNA was accomplished using the same method at a final concentration of $50 \mathrm{nM}$ of the siRNA duplex.

\section{Protein Collection, Immunoprecipitation and Western Blotting}

Following all cell treatments, media was aspirated from wells, and cells were collected in ice-cold phosphate-buffered saline (PBS). The cell pellet was then lysed in $100 \mu \mathrm{L}$ of cold radioimmunoprecipitation assay (RIPA) buffer containing $50 \mathrm{mM}$ Tris (pH 7.4), $150 \mathrm{mM} \mathrm{NaCl}, 0.5 \%$ sodium deoxycholate, $1 \%$ Nonidet P-40, 0.1\% Sodium dodecylsulfate (SDS), and a Roche COMPLETE protease inhibitor tablet. Samples were nutated for $30 \mathrm{~min}$ at $4^{\circ} \mathrm{C}$ with BSA-treated sepharose beads to remove cellular debris and centrifuged for $10 \mathrm{~min}$ to isolate the protein-rich supernatant. For co-immunoprecipitation (Co-IP), samples were gently incubated with relevant antibodies at $4^{\circ} \mathrm{C}$ for $30 \mathrm{~min}$ to precipitate each protein of interest. This was followed by the addition of protein A sepharose beads and an overnight incubation under the same conditions. The next day, samples were rinsed 3 times with RIPA buffer. Both western blot and Co-IP samples were then incubated at $65^{\circ} \mathrm{C}$ for $5 \mathrm{~min}$ prior to resolution using SDS-PAGE. Proteins were then transferred to nitrocellulose membranes, blocked with $5 \%$ milk powder in Tris-buffered saline (TBS), and shaken overnight at $4^{\circ} \mathrm{C}$ in milk containing the relevant primary antibody. The next day, membranes were washed

Fig. 1. Validation of Hypoxic Conditions. PC3 cells were incubated in normoxic or hypoxic conditions overnight, as described in the methods section. A Western blot of lysed cells in both conditions was performed using anti-Hif1alpha and control beta-actin antibodies.

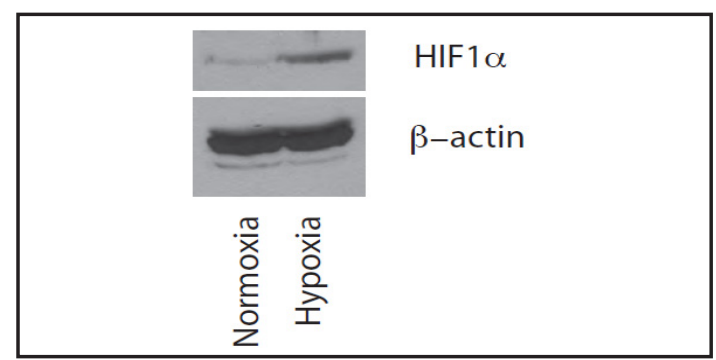




\section{Cellular Physiology Cell Physiol Biochem 2015;37:2071-2084

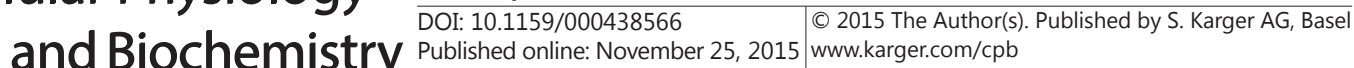 \\ Young et al.: SOD1 Regulation of CXCR4 Signaling}

with TBS containing 0.1\% Tween-20 and incubated with HRP-conjugated secondary antibodies for $1 \mathrm{hr}$ in TBS milk. Following another wash with TBS-Tween, membranes were visualized with Western Lightning Plus-ECL Chemiluminescence Substrate. ImageJ 4.3 software (NIH) was used to calculate immunoblot band densities.

\section{GST-Pulldown}

Pulldowns were performed as follows: CXCR4 IL1 was cloned into the pGEX4T1 vector. The fusion proteins were produced in BL21 Escherichia coli strain (New England Biolabs, Whitby, ON, Canada) according to the manufacturer's instructions. Gluthatione-Sepharose 4B (GE Healthcare, Baie d'Urfé, QC, Canada) was used for protein purification, and the purified recombinant proteins were analyzed by SDS-PAGE followed by Coomassie brilliant blue staining. A $15 \mu \mathrm{g}$ sample of GST-fused protein CXCR4 IL1 was then incubated with $15 \mu \mathrm{g}$ of recombinant SOD1 in a buffer composed of $150 \mathrm{mM} \mathrm{NaCl}, 10 \mathrm{mM}$ Tris-HCl, $1 \mathrm{mM}$ EDTA, $10 \%$ glycerol, $0.05 \%$ NP-40 and protease inhibitors. The incubated beads were washed three times with ice-cold PBS. Beads were then resuspended with Laemmli Buffer and boiled 2 min before loading on a 12\% SDSPAGE gel to detect expression.

\section{Annexin V/Propidium Iodide Staining}

In order to measure the extent of apoptosis in PC3 cells, cells were transfected with CXCR4 and SOD1 or SOD1 siRNA. After 24 hours, these cells were treated with etoposide $(50 \mathrm{ng} / \mathrm{ml})$ for 24 hours, to induce apoptosis. Following 6 hrs of etoposide treatment, cells were either stimulated with $100 \mathrm{ng} / \mathrm{mL}$ CXCL12 or with vehicle. Incubation took place under either normoxic or hypoxic cell culture conditions beginning at the time of stimulation. Cells were harvested and washed with PBS, then resuspended in Annexin V Assay Buffer as prescribed by the manufacturer's instructions. Cells were gently shaken in the dark with propidium iodide (PI) and Annexin V-FITC-conjugated stain for $20 \mathrm{~min}$. Cells were then examined by fluorescence microscopy and at least 5 fields of view were recorded using an Olympus IX81 microscope equipped with a Photometrics coolSNAP HQ2 camera and an Excite series 120Q light source. Annexin V stain was excited at $488 \mathrm{~nm}$ and images were captured at $525 \mathrm{~nm}$. PI was excited at $535 \mathrm{~nm}$. Rates of apoptosis were determined by dividing the number of cells that stained positive for Annexin $\mathrm{V}$ divided by the total number of cells.

\section{Transwell Migration Assays}

PC3 cells were transfected with HA-CXCR4 and SOD1 or SOD1 siRNA in 6-well plates. The growth media was aspirated and replaced with FBS-free media 24 hours post-transfection. After an additional 24 hours, cells were collected, rinsed with PBS and counted. Cultures were prepared such that $5.0 \times 10^{5}$ cells/ condition were resuspended in DMEM and plated into the top portion of a transwell migration plate that contains a polycarbonate membrane with a pore size of $5.0 \mu \mathrm{m}$ (Costar, Corning, NY). In the bottom portion of the well, $600 \mu \mathrm{L}$ of either DMEM or DMEM containing $100 \mathrm{ng} / \mathrm{mL}$ of CXCL12 was added. Cells were incubated for 24 hours under normoxic or hypoxic conditions. For quantification, membranes were rinsed with cold PBS and fixed in 100\% ice cold methanol for $15 \mathrm{~min}$ at room temperature. Fixed membranes were then stained with $0.5 \%$ crystal violet stain for $5 \mathrm{~min}$ to allow visualization of the cells. Non-migrated cells were then gently removed from the upper side of the membrane with a cotton bud. Membranes were rinsed in $\mathrm{dH}_{2} \mathrm{O}$ until the water ran clear, allowed to dry, and then mounted on a slide. At least 3 areas of the membrane were viewed under $10 \mathrm{x}$ objective of an Olympus IX81 and the number of cells for each field of view were counted. Net migration was determined by comparing the number of cells that migrated with the chemoattractant to the number of cell that migrated under control conditions.

\section{Statistical Analysis}

Statistical analysis was completed using GraphPrism software. All error bars are representative of mean +/-SEM. Two-tailed, unpaired student's t-tests were performed for analysis of endogenous CXCR4 and SOD1 levels, and co-IP data. Raw experimental data for Annexin V assays, p-AKT western blot data, and migration data was analyzed with a two-way ANOVA and a Bonferroni post-test. Annexin V data representing the fold change in apoptosis following stimulation was analyzed with a one-way ANOVA with a Bonferroni posttest. P-values are reported as follows: ${ }^{*} \mathrm{p}<0.05$, ${ }^{* *} \mathrm{p}<0.01,{ }^{* * *} \mathrm{p}<0.001$.

\section{KARGER}




\section{Cellular Physiology Cell Physiol Biochem 2015;37:2071-2084

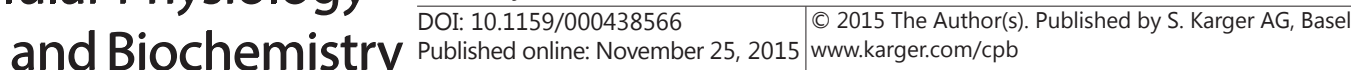 \\ Young et al.: SOD1 Regulation of CXCR4 Signaling}

\section{Results}

Expression of Endogenous and Transfected CXCR4 and SOD1 in PC3 cells

To evaluate the potential effects of a protein-protein interaction between CXCR4 and SOD1, we first needed to verify the presence of these molecules in PCa cells. We chose to use the PC3 cells for several reasons: PC3 cells have been reported to express endogenous levels of CXCR4 [22], are more invasive in comparison with other PCa cells lines as measured in vitro using matrigel assays [23], and are derived from bone marrow metastases, characteristic of the advanced metastatic form of the disease that we are investigating [24].

PC3 cells were maintained in either normoxic or hypoxic cell culture conditions, lysed, and immunoblotted for the presence of both SOD1 and CXCR4. Fig. 2A and Fig. 2B show that both SOD1 and CXCR4, respectively, are expressed endogenously in PC3 cells under both normal and hypoxic conditions. The histograms in Fig. 2A and 2B indicate that that SOD1 levels increase approximately 1.7 -fold following an $8 \mathrm{hr}$ treatment in hypoxia and CXCR4 levels increase similarly, but with a higher degree of variability. siRNA-mediated knockdown resulted in lower levels of SOD1 in these cells as shown by western blot against SOD1 (Fig. 2C). Results show that there was approximately 50\% reduction in SOD1 expression following SOD1 siRNA transfection.

Fig. 2. Endogenous Levels of CXCR4 and SOD1 in Normal and Hypoxic Conditions in PC3 Cells. (A) Top panel shows a representative western blot against SOD1 (A) or CXCR4 (B) under normoxic and hypoxic conditions with $\beta$-actin as loading control. Bottom panels summarize the results of the western blot experiment. (C) Representative blot and histogram showing the effect of specific and non-specific siRNAs on the expression of SOD1 or $\beta$-actin in PC3 cells. Results represent the means \pm SEM of 5 independent experiments. * $p<0.05$, using two-tailed unpaired Student's $t$ test.

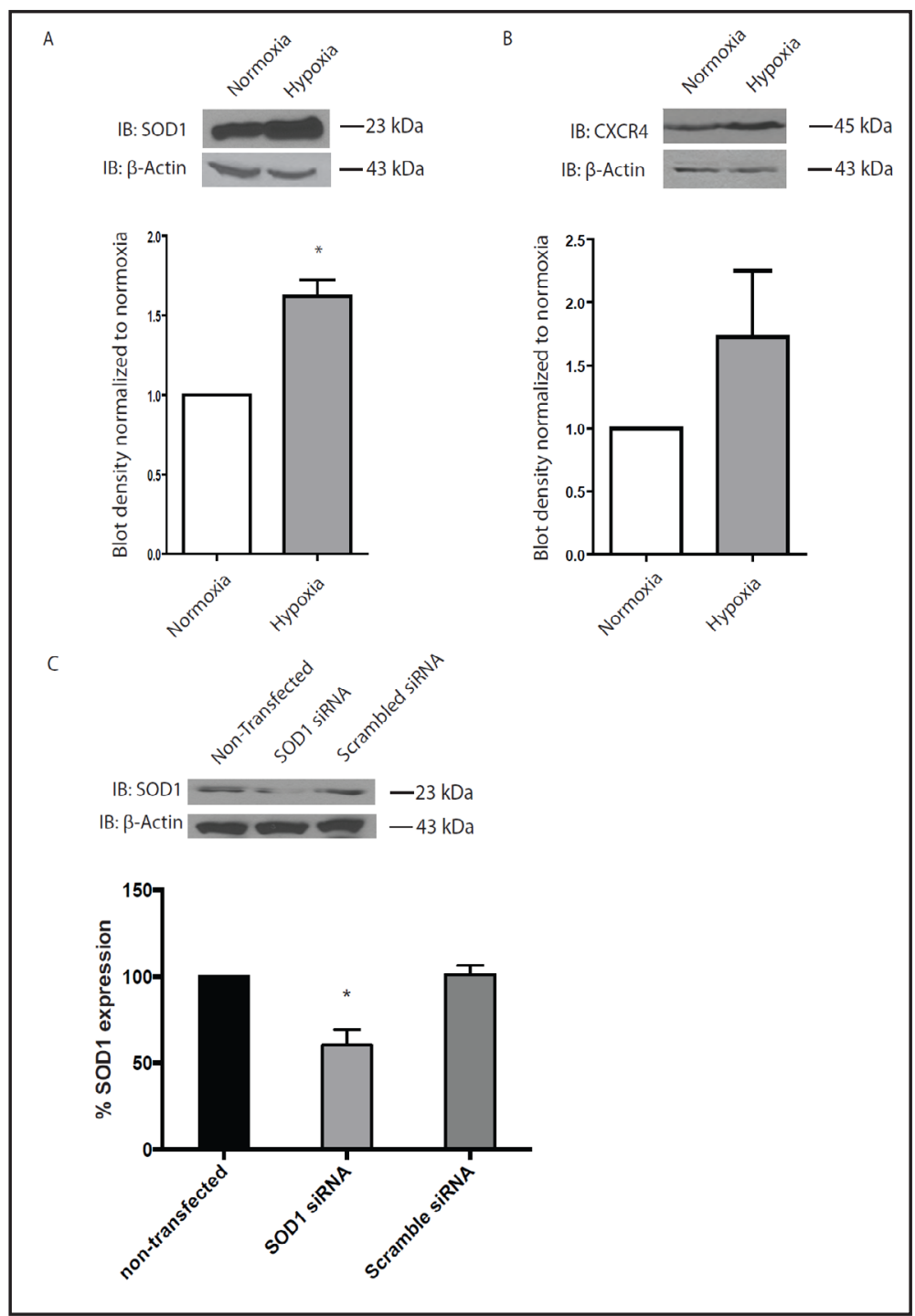




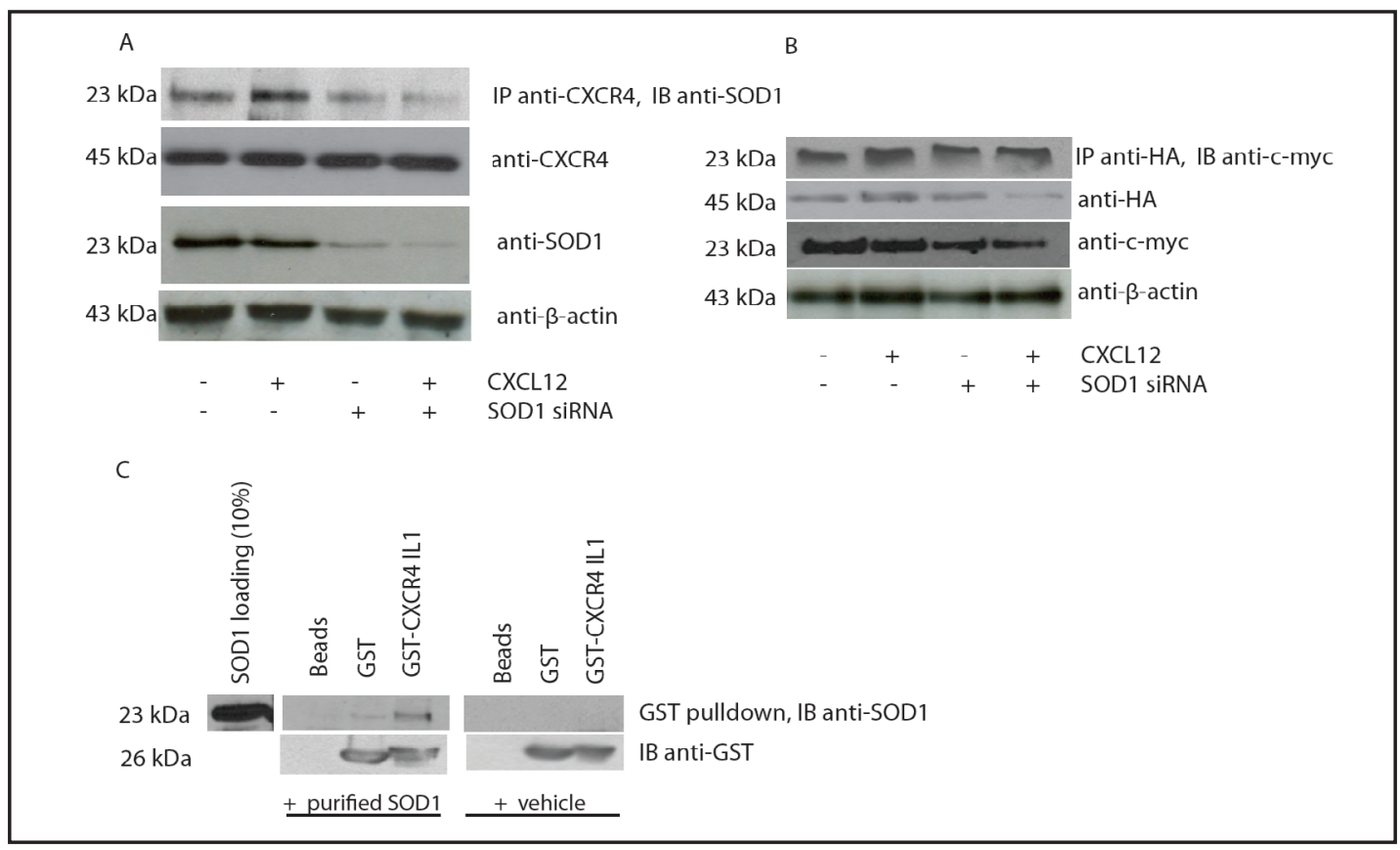

Fig. 3. Interaction between CXCR4 and SOD1 in PC3 Cells. (A) Co-immunoprecipitation of endogenous CXCR4 and SOD1 in PC3 cells and the effect of the SOD1 siRNA. (B) Co-IP of overexpressed HA-tagged CXCR4 and c-Myc-tagged SOD1. (C) GST-pulldown of recombinant SOD1 with GST-CXCR4 ICL1 WT. Top panel: Immunoblot of SOD1 following pulldown or in absence of pulldown. Lanes 2-4 show the pulldown with empty beads, GST alone or CXCR4 ICL1, respectively in presence of purified recombinant SOD1. Lane 4 shows the direct interaction of ICL1 with SOD1. In lanes 5-7, no recombinant SOD1 was added to the reaction. Lane 1 is purified recombinant SOD1 alone, where $10 \%$ of the pulldown reaction was loaded onto the gel. Bottom panel: Immunoblot of GST constructs expressed in the top panel. Results are representative of 4 independent experiments.

Fig. 4. Interaction Levels between CXCR4 and SOD1 in PC3 cells in both Normal and Hypoxic Conditions. (A) Representative results of a co-immunoprecipitation experiment under normoxic and hypoxic conditions. The second and fourth lanes show results following stimulation with $100 \mathrm{ng} / \mathrm{ml}$ CXCL12. Top panel shows the results of the Co-IP, where CXCR4 was precipitated and SOD1 was immunoblotted. (B) Histogram summarizing the results of the co-IP experiment seen in A. Results represent the means \pm SEM of 4 independent experiments. ${ }^{*} p<0.05$, using two-way ANOVA with a Bonferroni post-test.

Endogenous Interaction of CXCR4 with SOD1 in PC3 cells in Normal and Hypoxic Conditions

Following the original indication that SOD1 was interacting with CXCR4 obtained using a yeast-two hybrid screen between CXCR4 intracellular loop 1 (ICL1) as bait and a bone marrow library (data not shown), we wanted to confirm the existence of a SOD1/

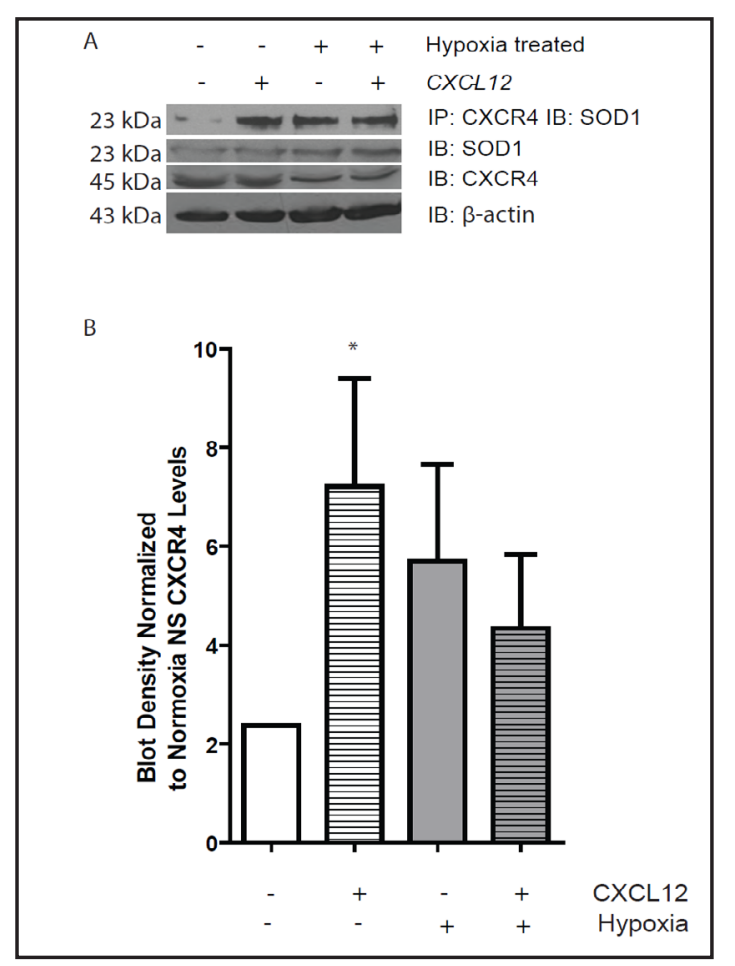




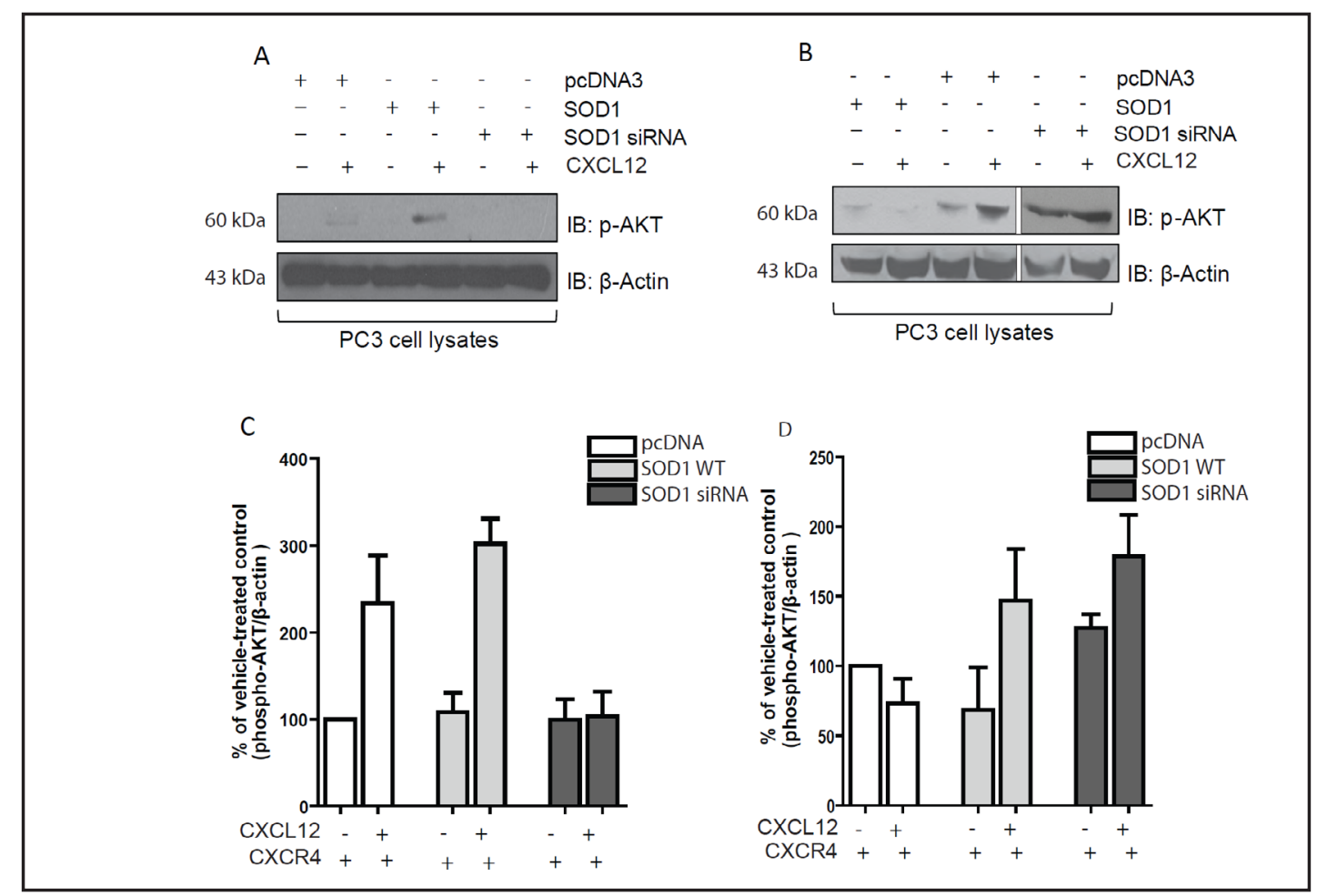

Fig. 5. Effects of SOD1 and Hypoxia on CXCR4-Mediated Akt Activation in PC3 Cells in both Normal and Hypoxic Conditions. (A) Representative western blot of p-Akt where PC3 cells were treated for 15 min with $100 \mathrm{ng} / \mathrm{ml} \mathrm{CXCL12}$ or water prior to cell lysis. (B) Representative western blot of p-Akt for cell lysates from PC3 cells maintained in hypoxic conditions for $8 \mathrm{hr}$ prior to stimulation. Histograms of p-Akt blot density normalized to $\beta$-actin in normoxia (C) or hypoxia (D) Results represent the means \pm SEM of 5 independent experiments under normoxic conditions, and 4 independent experiments under hypoxic conditions. ${ }^{*} p<0.05,{ }^{* *} \mathrm{p}<0.01$, using two-way ANOVA with a Bonferroni post-test.

CXCR4 complex in living cells. PC3 cells treated with CXCL12 or vehicle were lysed, and subjected to immunoprecipitation of CXCR4 and immunoblotting of SOD1. Results displayed in Fig. 3A (lanes 1 and 2) demonstrate that endogenous CXCR4 and SOD1 interact in PC3 cells under normoxic conditions. This interaction was affected by the receptor's endogenous ligand, as $15 \mathrm{~min}$ stimulation with $100 \mathrm{ng} / \mathrm{mL}$ CXCL12 increased the interaction levels, as measured by co-IP. Expression of a SOD1 siRNA decreased the extent of the interaction between the two proteins. Co-IP of SOD1 with CXCR4 was confirmed using HA-tagged CXCR4 and myc-tagged SOD1 as shown in Fig. 3B. Finally, to demonstrate that this interaction is direct, a GST-pulldown using the ICL1 of CXCR4 as the purified GST-construct, and recombinant SOD1 (Fig. 3C) was performed. Our results indicate a direct interaction between CXCR4 ICL1 with SOD1.

Next, we tested the effect of hypoxia on the protein-protein interaction between CXCR4 and SOD1. As can be seen in Fig. 4A and the corresponding histogram in Fig. 4B, there is basal level of interaction between these proteins under both normal and hypoxic conditions. Following stimulation with $100 \mathrm{ng} / \mathrm{mL}$ CXCL12 for $15 \mathrm{~min}$ in normoxic conditions, this interaction increased significantly; however, when the cells were maintained under hypoxia for $8 \mathrm{hr}$ prior to stimulation, similar interaction levels between CXCR4 and SOD1 were observed and CXCL12 stimulation no longer had an effect on this interaction.

Modulation of CXCR4 Signal Transduction by SOD1

Due to the importance of CXCR4-activated signaling pathways in the metastatic transition, we next decided to determine if SOD1 levels affected CXCR4 signal transduction. 


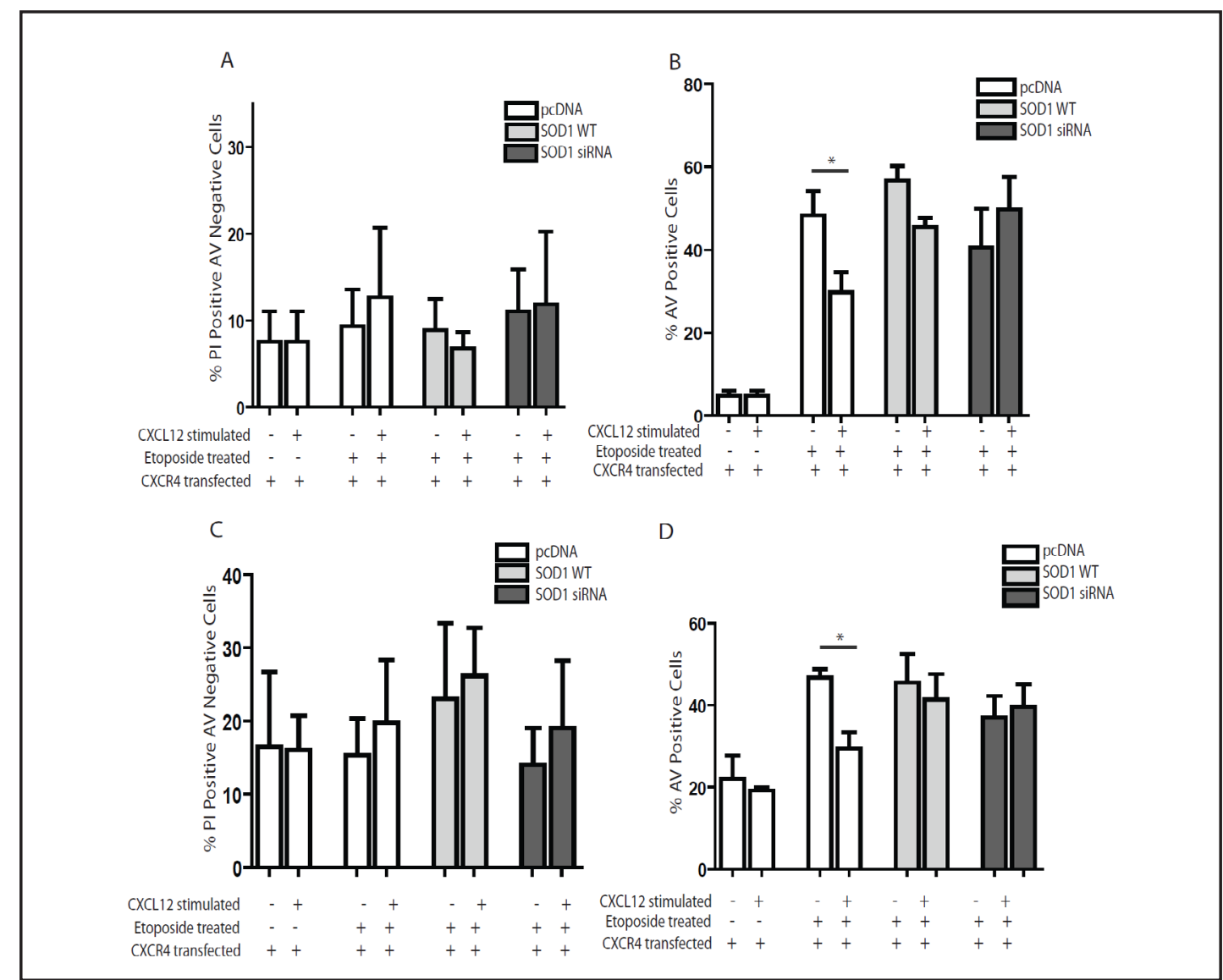

Fig. 6. Effect of SOD1 and CXCR4 on Apoptosis Levels in PC3 Cells in Normoxic or Hypoxic Conditions. (A) Necrotic cells, identified as propidium iodide (PI) positive and Annexin V (AV) negative in normoxia. Etoposide was used at a concentration of $50 \mathrm{ng} / \mathrm{ml}$ for $24 \mathrm{hr}$ to induce apoptosis in these cells. $100 \mathrm{ng} / \mathrm{ml} \mathrm{CXCL12}$ was used to stimulate PC3 cells for $18 \mathrm{hr}$ prior to experimentation. (B) Apoptotic cell percentage identified as positive for AV staining in normoxia. (C) Necrotic cells, when maintained under hypoxic conditions, beginning with stimulation. (D) Apoptotic cells maintained under hypoxic conditions. Results represent the means \pm SEM of 4 independent experiments in hypoxic conditions. ${ }^{*} p<0.05$, using two-way ANOVA with a Bonferroni post-test.

Since several signaling pathways are activated by CXCR4, we chose to measure AKT activation due to its established role in cell survival and migration, processes important during cancer progression [15].

AKT is phosphorylated following activation of PI3K by either receptor tyrosine kinases or GPCRs at the cell surface. Active p-AKT is then able to reduce apoptosis by inhibiting proapoptotic proteins such as BAD, or by activating anti-apoptotic factors such as NF- $\kappa B[25,26]$. We measured the levels of p-AKT in PC3 cells following activation of CXCR4 by $100 \mathrm{ng} / \mathrm{mL}$ CXCL12 for $15 \mathrm{~min}$ and the results are shown in Fig. 5. Representative western blots of p-AKT using cells maintained under normoxic conditions are shown in Fig. 5A, and a corresponding histogram showing the change in AKT activation is shown in Fig. 5C. Our results show that cells overexpressing SOD1 showed a significant increase in AKT activation following stimulation with CXCL12 and that no increase in p-AKT levels was observed following stimulation in cells where SOD1 was knocked down. Under hypoxic conditions, no significant increase in AKT activation was observed following CXCR4 stimulation with CXCL12, regardless of SOD1 expression levels, again suggesting that SOD1 does not participate in these events under hypoxic conditions (Fig. 5B and 5D).

\section{KARGER}




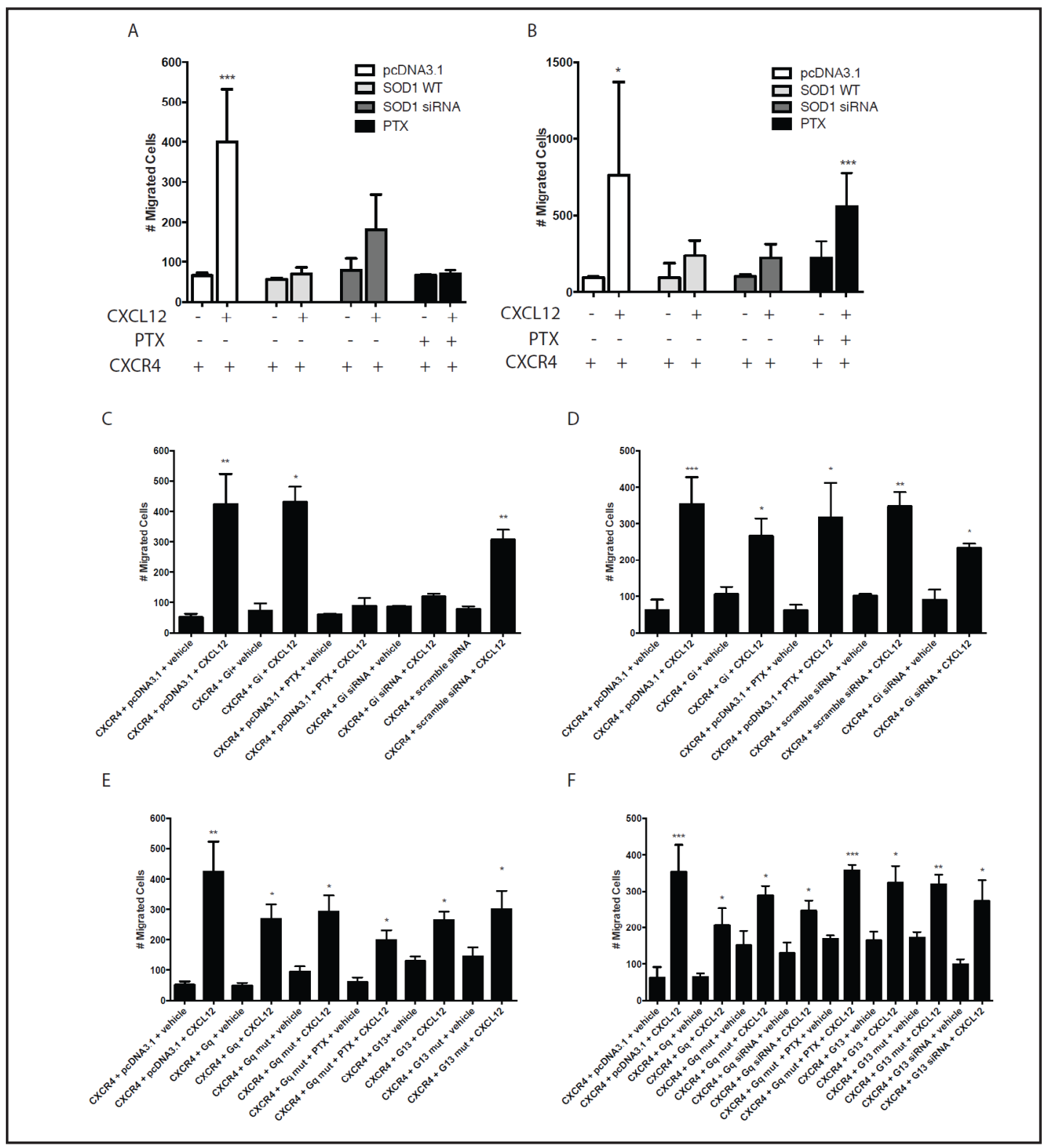

Fig. 7. Effect of SOD1 and Pertussis Toxin on CXCR4-Mediated Migration in Response to CXCL12 in both Normoxic and Hypoxic Conditions. Histograms summarizing transwell migration results using PC3 cells transfected with CXCR4 under normoxic conditions (A) and hypoxic conditions (B). Effect of G $\alpha_{i}$ on the migration of PC3 cells towards CXCL12 or media containing equivalent volume of ethanol (vehicle) in normoxia (C) and hypoxia (D). Effect of PTX-insensitive G proteins on the migration of PC3 cells towards CXCL12 or its vehicle in normoxia (E) and hypoxia (F). Results represent the means \pm SEM of at least 3 independent experiments. ${ }^{*} p<0.05,{ }^{* *} p<0.01,{ }^{* * *} p<0.001$, using two-way ANOVA with a Bonferroni post-test.

\section{Effect of SOD1 on etoposide-induced apoptosis}

It is well known that many types of cancer arise and progress due to dysregulation of apoptotic pathways, resulting in aberrant cell growth and survival. Fig. 6 shows that following treatment with etoposide under normoxic conditions, an apoptosis-inducing topoisomerase inhibitor, there is no difference in levels of necrosis, as measured by percentage of PI positive nuclei and Annexin V negative cells. No significant effect of CXCL12 stimulation or SOD1 expression level on necrosis levels was observed either (Fig. 6A). Upon etoposide treatment, apoptosis levels increase significantly (Fig. 6B); an effect that was partially blocked with 


\section{Cellular Physiology Cell Physiol Biochem 2015;37:2071-2084

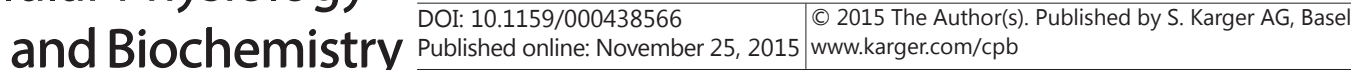 Young et al.: SOD1 Regulation of CXCR4 Signaling}

Fig. 8. Validation of G-Protein Isoforms siRNAs. PC3 cells were transfected with a G protein isoform tagged with Rluc and their specific siRNA or scramble siRNA (Qiagen). Results indicate that each siRNA is very efficient at reducing their target G protein expression. Results are representative of 3 independent experiments.

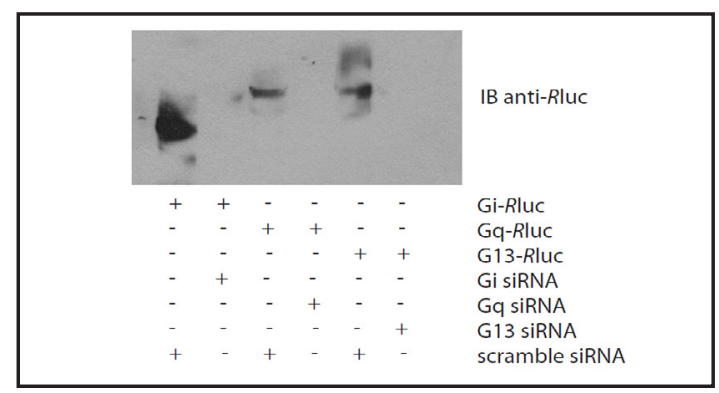

overnight treatment with $100 \mathrm{ng} / \mathrm{mL}$ CXCL12 of pcDNA transfected cells. The same trend was observed following CXCL12 treatment when SOD1 was overexpressed, while no reduction was present when SOD1 siRNA was expressed. Under hypoxic conditions (Fig. 6C), higher levels of necrosis were observed (approximately 15-25\%, instead of 8-12\% in normoxia), which none of the treatments altered. Hypoxic conditions increased the overall basal apoptosis levels, without etoposide treatment, to approximately $20 \%$ of all cells and while pcDNA expressing cells saw a decrease in apoptosis following CXCL12 treatment, SOD1 WT or siRNA overexpression did not induce any change in apoptosis measurements (Fig. 6D).

\section{Effects of SOD1 on CXCL12-Mediated Cell Migration and G protein coupling}

We next measured the number of cells that migrated in response to exposure to CXCL12 using a transwell migration assay. Following overnight exposure to $100 \mathrm{ng} / \mathrm{mL}$ CXCL12, there was a significant increase in migration in cells transfected with pcDNA (Fig. 7A). However, cells transfected with SOD1 showed a near-complete loss of CXCL12-induced migration. When SOD1 siRNA was co-expressed, a partial rescue of migration was observed. When performed under hypoxic conditions (Fig. 7B), we detected no difference between SOD1 WT overexpression or knockdown suggesting that other mechanisms might be at play when SOD1 levels are changed under hypoxic conditions. However, pcDNA transfected cells do show a significant increase in migration following stimulation.

$\mathrm{G}_{\mathrm{i}}$-coupled GPCRs, like CXCR4, are linked to cell migration towards chemotactic signals. Thus, we tested the effect of the $G_{i}$ inhibitor pertussis toxin (PTX) on the migration of PC3 cells. In normoxia, PTX treatment abolished migration of PC3 cells (Fig. 7C) while it has less effect on CXCL12-induced chemotaxis in hypoxia (Fig. 7D). The dependence on $\mathrm{G}_{\mathrm{i}}$ is confirmed in normoxia (Fig. 7C) using siRNA (validated in Fig. 8) directed against $\mathrm{G}_{\mathrm{i}}$, but not in hypoxic conditions (Fig. 7D). Given the differences observed with PTX treatment, we further investigated the potential role of non-PTX-sensitive $G$ proteins in the migration process as it was previously shown that $G$ proteins $G_{q}$ and $G_{13}$ (both PTX-insensitive) may also be involved in CXCL12/CXCR4-dependent migration under some circumstances [27, 28]. Expression of either G protein specific siRNAs, their scramble siRNA control, or functionally active xanthine nucleotide-specific $\mathrm{G} \alpha$ that act as dominant-negative proteins were used to dissect specificities of receptor/G-protein coupling [29]. Our results indicate that neither $\mathrm{G}_{\mathrm{q}}$, nor $\mathrm{G}_{13}$ contribute to PC3 migration induced by CXCL12 (Fig. 7E; normoxia, Fig. 7F; hypoxia). Our results suggest that CXCL12-induced migration of PC3 cells in hypoxia is either G proteinindependent, or relies on a non-canonical CXCR4-coupled G protein isoform.

\section{Discussion}

A plethora of evidence implicates CXCR4 in the development, progression and metastatic potential of prostate cancer [1-4, 30-33]. Our lab performed a yeast-two hybrid screen to find proteins that interact with CXCR4 in an attempt to understand regulation of this GPCR. This screen found that CXCR4 interacted with the antioxidant enzyme SOD1. Due to the role that CXCR4 and SOD1 play independently in prostate cancer incidence and metastasis, this study aimed to examine the effects of the interaction between CXCR4 and SOD1. As we were primarily interested in the metastatic process, we examined signaling pathways known to 
play a role in survival and proliferation, cell migration and apoptosis, all processes known to be important for cancer spread. The results of our study demonstrate that SOD1 interacts with CXCR4 in a PCa cell line and that the modulation of SOD1 levels can alter CXCR4 signaling.

\section{CXCR4 and SOD1 direct interaction}

Clontech's Matchmaker Gold Yeast Two-Hybrid System (Y2H), a stringent four-reporter system that leads to a high yield of true positive interactions, was used to identify interaction partners of CXCR4. The intracellular loops and C-tail of CXCR4 were used to probe a fulllength cDNA library (data not shown), generating a positive interaction between CXCR4 ICL1 and SOD1. To confirm this interaction, we assessed the interaction of endogenous CXCR4 and SOD1. CXCR4 is well known to be involved in cancer metastasis and SOD1 polymorphisms are linked to PCa aggressiveness [34], and SOD1 inhibitors have been shown to inhibit proliferation and tumour growth [21]. These findings suggest that the interaction of CXCR4 and SOD1 may be important in regulating cancer progression. The results from our co-immunoprecipitations, combined with the GST-pulldown between CXCR4 ICL1 and recombinant SOD1 suggest a new interacting partner for ICL1 that may play a role in the regulation of GPCR signalling.

\section{SOD1 May Modulate CXCR4-Induced signal transduction}

The PI3K/AKT pathway is closely linked to cell survival and increases in both cell size and number [26]. Studies have demonstrated that there is an increase in active, p-AKT following stimulation with dihydrotestosterone in PCa cells cultured under normoxic conditions [35]. Our results show activation of AKT following CXCL12 stimulation. This result supports earlier findings, where stimulation of CXCR4 causes the phosphorylation of AKT in a variety of cancer cell types [26,36]. Overexpression of SOD1 did not change this result. However, cells transfected with SOD1 siRNA displayed a blockade of CXCL12-induced AKT phosphorylation. Taken together, these results suggest that SOD1 may be important for CXCR4-mediated activation of AKT under normoxic conditions and that the endogenous SOD1 in PC3 cells may be sufficient to allow AKT activation. Under hypoxic conditions, there was no significant activation of AKT following stimulation, regardless of SOD1 expression levels. Therefore, the CXCR4-induced phosphorylation of AKT that could lead to protection of PC3 cells against apoptosis is no longer present under hypoxic conditions.

SOD1 Protects Against Apoptosis under Normoxic, but not Hypoxic Conditions

There is a wealth of evidence available to suggest that CXCR4 stimulation decreases apoptosis [33, 37, 38]. Our results show that PC3 cells transfected with CXCR4 exhibited a significant decrease in etoposide-induced apoptosis following $18 \mathrm{hr}$ treatment with CXCL12. SOD1 overexpressing cells trended towards a decrease in apoptosis levels following CXCL12 stimulation. This finding is in line with what has been seen previously, as antioxidants in general have anti-apoptotic effects through their ability to scavenge ROS [39]. However, while there was a trend towards a decrease in apoptosis following stimulation of SOD1 overexpressing cells in normoxia, this was no longer apparent under hypoxic conditions. Taken together, our results suggest that SOD1 protects cells from apoptosis in normoxic, but not necessarily in hypoxic environments.

\section{SOD1 effect on CXCL12-induced Migration}

CXCR4 activation has been shown to cause cell migration $[1,6,13,36,40]$. Our results showed that SOD1 overexpression decreased CXCL12-induced migration in normoxia. This finding is agreement with those found by Chetram et al. [25], where cells exhibited higher levels of CXCR4-mediated migration following the addition of $\mathrm{H}_{2} \mathrm{O}_{2}$, mimicking ROS accumulation. Cells transfected with SOD1 siRNA displayed partial rescue of migration in response to CXCL12. Under hypoxic conditions, PC3 cells showed a significant increase in migration following CXCL12 stimulation with overexpressed CXCR4. However, neither SOD1 overexpressing cells nor the SOD1 siRNA treated cells exhibited the same increase in migration following stimulation. Yamasaki et al. [35] examined the differential response 
of PCa cells to acute versus chronic hypoxic exposure, where chronic exposure referred to cells maintained in $1 \% \mathrm{O}_{2}$ for 6 months. Their findings suggested that acute hypoxia caused a decrease in cell migration, while chronic hypoxia resulted in an increase in migration and invasion. It has been demonstrated that $\mathrm{G} \alpha_{i}$ signaling is important for CXCL12-mediated migration, while $\mathrm{G} \alpha$ is involved in continuous inhibition of migration in $\mathrm{T}$ cells [41]. Pertussis toxin (PTX) was used in order to determine whether or not CXCR4-mediated migration of PC3 cells was dependent on G $\alpha_{i}$ Under normoxic conditions, PTX inhibited CXCL12-stimulated migration of PC3 cells. This finding is in support of other studies that noted PTX significantly impaired CXCR4-mediated migration of cancer cells [42, 43]. In contrast, under hypoxic conditions, PTX-treated cells still showed a significant increase in migration following stimulation with CXCL12. This finding suggested that the migration of PC3 cells in response to CXCL12 under hypoxic conditions is not dependent on G $\alpha_{i^{\circ}}$ CXCR4 couples with both PTX-sensitive and -insensitive G proteins, and it has been suggested that the balance between $\mathrm{G} \alpha_{i}$ and $\mathrm{G} \alpha$ signaling is important for the regulation of cell migration [41]. In addition to $\mathrm{G \alpha}_{\mathrm{i}}$ and $\mathrm{G} \alpha_{\mathrm{q}}$ CXCR4-mediated chemotaxis and transendothelial migration of metastatic breast cancer cells was shown to require activation of $\mathrm{G \alpha}_{13}$. Whereas most of the physiological functions of CXCR4 involve activation of $\mathrm{G} \alpha_{i}$, it is becoming apparent that CXCR4 can interact physically with other $G$ proteins including $G \alpha_{q}$ and $G \alpha_{13}$ [27]. Our studies showed that under normoxic conditions, $\mathrm{G} \alpha_{i}$ is involved in migration. However, under hypoxic conditions, blockade of $\mathrm{G} \alpha_{i}, \mathrm{G} \alpha_{q}$ or $\mathrm{G \alpha} \alpha_{13}$ did block migration, suggesting that other mechanisms are likely involved in CXCL12/CXCR4-regulated migration under certain conditions, such as G-protein independent pathways or non-canonical G proteins.

Considering our results, we propose that regulation of CXCR4 signaling by SOD1 is possible under normoxic conditions, where low levels of ROS are present in the cell, and where SOD1 interacts with CXCR4 to regulate its signaling. Under hypoxic conditions, where ROS levels may increase in the cell, SOD1 reacts to oxidative stress. SOD1 loses the ability to regulate CXCR4 signal transduction, whereby a decrease in protection against apoptosis and dysregulation of G protein signaling is observed. Our results highlight a new potential mechanism by which the influence of the cell environment, here oxidative stress, could be integrated by a sensor (SOD1) to influence cell fate by regulating CXCR4 survival and migration signaling pathways. Thus, the interaction between CXCR4 and SOD1 could represent a new pharmacological target to potentially reduce cancer cell migration.

\section{Abbreviations}

Bioluminescence resonance energy transfer (BRET); bovine serum albumin (BSA); coimmunoprecipitation (Co-IP); C-X-C motif chemokine 12 (CXCL12)/Stromal cell-derived factor 1 alpha (SDF1 $\alpha$ ); C-X-C chemokine receptor type 4 (CXCR4); Dulbecco's Modified Eagle's medium (DMEM); bovine serum (FBS); G protein-coupled receptor (GPCR); horseradish peroxidase (HRP); phosphate buffered saline (PBS); prostate cancer (PCa); propidium iodide (PI); phosphoinositide 3-kinase (PI3K); Pertussis toxin (PTX); Renilla luciferase (Rluc); radioimmunoprecipitation assay (RIPA); reactive oxygen species (ROS); sodium dodecylsulfate (SDS); Small-Interfering RNA (siRNA); superoxide dismutase (SOD); tris-buffered saline (TBS); yellow fluorescent protein (YFP).

\section{Acknowledgements}

We thank Dr. Terence Hébert for critical reading of the manuscript drafts. This work was partially supported by grants from NSERC [RGPIN/355310-2013] and Dalhousie University Faculty of Medicine Bridge funding to D.J.D.

\section{Disclosure Statement}

The authors declare no financial or commercial conflict of interest.

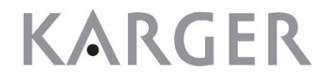




\section{Cellular Physiology Cell Physiol Biochem 2015;37:2071-2084

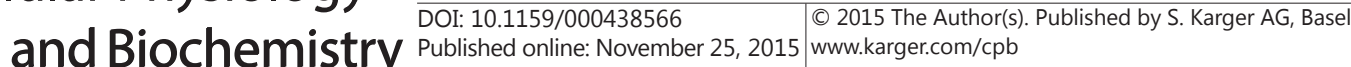 \\ Young et al.: SOD1 Regulation of CXCR4 Signaling}

\section{References}

1 Zhang S, Qi L, Li M, Zhang D, Xu S, Wang N, Sun B: Chemokine cxcl12 and its receptor cxcr4 expression are associated with perineural invasion of prostate cancer. J Exp Clin Cancer Res 2008;27:62.

2 Akashi T, Koizumi K, Tsuneyama K, Saiki I, Takano Y, Fuse H: Chemokine receptor cxcr4 expression and prognosis in patients with metastatic prostate cancer. Cancer Sci 2008;99:539-542.

3 Taichman RS, Cooper C, Keller ET, Pienta KJ, Taichman NS, McCauley LK: Use of the stromal cell-derived factor-1/cxcr4 pathway in prostate cancer metastasis to bone. Cancer Res 2002;62:1832-1837.

4 Sun YX, Wang J, Shelburne CE, Lopatin DE, Chinnaiyan AM, Rubin MA, Pienta KJ, Taichman RS: Expression of cxcr4 and cxcl12 (sdf-1) in human prostate cancers (pca) in vivo. J Cell Biochem 2003;89:462-473.

5 Dontu G, Al-Hajj M, Abdallah WM, Clarke MF, Wicha MS: Stem cells in normal breast development and breast cancer. Cell Prolif 2003;36:59-72.

6 Muller A, Homey B, Soto H, Ge N, Catron D, Buchanan ME, McClanahan T, Murphy E, Yuan W, Wagner SN, Barrera JL, Mohar A, Verastegui E, Zlotnik A: Involvement of chemokine receptors in breast cancer metastasis. Nature 2001;410:50-56.

7 Geminder H, Sagi-Assif O, Goldberg L, Meshel T, Rechavi G, Witz IP, Ben-Baruch A: A possible role for cxcr4 and its ligand, the cxc chemokine stromal cell-derived factor-1, in the development of bone marrow metastases in neuroblastoma. J Immunol 2001;167:4747-4757.

8 Porcile C, Bajetto A, Barbero S, Pirani P, Schettini G: Cxcr4 activation induces epidermal growth factor receptor transactivation in an ovarian cancer cell line. Ann NY Acad Sci 2004;1030:162-169.

9 Hall JM, Korach KS: Stromal cell-derived factor 1, a novel target of estrogen receptor action, mediates the mitogenic effects of estradiol in ovarian and breast cancer cells. Mol Endocrinol 2003;17:792-803.

10 Libura J, Drukala J, Majka M, Tomescu 0, Navenot JM, Kucia M, Marquez L, Peiper SC, Barr FG, JanowskaWieczorek A, Ratajczak MZ: Cxcr4-sdf-1 signaling is active in rhabdomyosarcoma cells and regulates locomotion, chemotaxis, and adhesion. Blood 2002;100:2597-2606.

11 Hirota K, Semenza GL: Regulation of angiogenesis by hypoxia-inducible factor 1. Crit Rev Oncol Hematol 2006;59:15-26.

12 Schioppa T, Uranchimeg B, Saccani A, Biswas SK, Doni A, Rapisarda A, Bernasconi S, Saccani S, Nebuloni M, Vago L, Mantovani A, Melillo G, Sica A: Regulation of the chemokine receptor cxcr4 by hypoxia. J Exp Med 2003;198:1391-1402.

13 Helbig G, Christopherson KW, 2nd, Bhat-Nakshatri P, Kumar S, Kishimoto H, Miller KD, Broxmeyer HE, Nakshatri H: Nf-kappab promotes breast cancer cell migration and metastasis by inducing the expression of the chemokine receptor cxcr4. J Biol Chem 2003;278:21631-21638.

14 Gillies K, Wertman J, Charette N, Dupre DJ: Anterograde trafficking of cxcr4 and ccr2 receptors in a prostate cancer cell line. Cell Physiol Biochem 2013;32:74-85.

15 Barbero S, Bonavia R, Bajetto A, Porcile C, Pirani P, Ravetti JL, Zona GL, Spaziante R, Florio T, Schettini G: Stromal cell-derived factor 1alpha stimulates human glioblastoma cell growth through the activation of both extracellular signal-regulated kinases 1/2 and akt. Cancer Res 2003;63:1969-1974.

16 Vlahakis SR, Villasis-Keever A, Gomez T, Vanegas M, Vlahakis N, Paya CV: G protein-coupled chemokine receptors induce both survival and apoptotic signaling pathways. J Immunol 2002;169:5546-5554.

17 Taniguchi N: Clinical significances of superoxide dismutases: Changes in aging, diabetes, ischemia, and cancer. Adv Clin Chem 1992;29:1-59.

18 Johnson F, Giulivi C: Superoxide dismutases and their impact upon human health. Mol Aspects Med 2005;26:340-352.

19 Nicco C, Laurent A, Chereau C, Weill B, Batteux F: Differential modulation of normal and tumor cell proliferation by reactive oxygen species. Biomed Pharmacother 2005;59:169-174.

20 Okada F, Shionoya H, Kobayashi M, Kobayashi T, Tazawa H, Onuma K, Iuchi Y, Matsubara N, Ijichi T, Dugas B, Hosokawa M: Prevention of inflammation-mediated acquisition of metastatic properties of benign mouse fibrosarcoma cells by administration of an orally available superoxide dismutase. Br J Cancer 2006;94:854862.

21 Lin J, Zahurak M, Beer TM, Ryan CJ, Wilding G, Mathew P, Morris M, Callahan JA, Gordon G, Reich SD, Carducci MA, Antonarakis ES: A non-comparative randomized phase ii study of 2 doses of atn-224, a copper/zinc superoxide dismutase inhibitor, in patients with biochemically recurrent hormone-naïve prostate cancer. Urol Oncol 2013;31:581-588.

22 Singh S, Singh UP, Stiles JK, Grizzle WE, Lillard JW: Expression and functional role of ccr9 in prostate cancer cell migration and invasion. Clin Cancer Res 2004;10:8743-8750. 


\section{Cellular Physiology Cell Physiol Biochem 2015;37:2071-2084

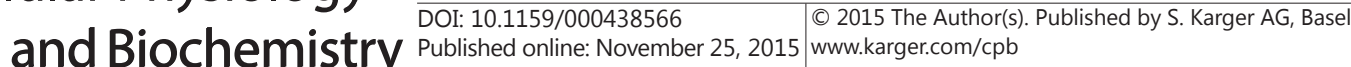 \\ Young et al.: SOD1 Regulation of CXCR4 Signaling}

23 Pulukuri SM, Gondi CS, Lakka SS, Jutla A, Estes N, Gujrati M, Rao JS: Rna interference-directed knockdown of urokinase plasminogen activator and urokinase plasminogen activator receptor inhibits prostate cancer cell invasion, survival, and tumorigenicity in vivo. J Biol Chem 2005;280:36529-36540.

24 Sobel RE, Sadar MD: Cell lines used in prostate cancer research: A compendium of old and new lines--part 1. J Urol 2005;173:342-359.

25 Chetram MA, Don-Salu-Hewage AS, Hinton CV: Ros enhances cxcr4-mediated functions through inactivation of pten in prostate cancer cells. Biochem Biophys Res Commun 2011;410:195-200.

26 Vivanco I, Sawyers CL: The phosphatidylinositol 3-kinase akt pathway in human cancer. Nat Rev Cancer 2002;2:489-501.

27 Yagi H, Tan W, Dillenburg-Pilla P, Armando S, Amornphimoltham P, Simaan M, Weigert R, Molinolo AA, Bouvier M, Gutkind JS: A synthetic biology approach reveals a cxcr4-g13-rho signaling axis driving transendothelial migration of metastatic breast cancer cells. Sci Signal 2011;4:ra60.

28 Soede RD, Wijnands YM, Kamp M, van der Valk MA, Roos E: Gi and gq/11 proteins are involved in dissemination of myeloid leukemia cells to the liver and spleen, whereas bone marrow colonization involves gq/11 but not gi. Blood 2000;96:691-698.

29 Gille A, Seifert R: Xanthine nucleotide-specific g-protein alpha-subunits: A novel approach for the analysis of g-protein-mediated signal transduction. Naunyn Schmiedebergs Arch Pharmacol 2004;369:141-150.

30 Darash-Yahana M, Pikarsky E, Abramovitch R, Zeira E, Pal B, Karplus R, Beider K, Avniel S, Kasem S, Galun E, Peled A: Role of high expression levels of cxcr4 in tumor growth, vascularization, and metastasis. FASEB J 2004;18:1240-1242.

31 Domanska UM, Timmer-Bosscha H, Nagengast WB, Oude Munnink TH, Kruizinga RC, Ananias HJ, Kliphuis NM, Huls G, De Vries EG, de Jong IJ, Walenkamp AM: Cxcr4 inhibition with amd3100 sensitizes prostate cancer to docetaxel chemotherapy. Neoplasia 2012;14:709-718.

32 Fischer T, Nagel F, Jacobs S, Stumm R, Schulz S: Reassessment of cxcr4 chemokine receptor expression in human normal and neoplastic tissues using the novel rabbit monoclonal antibody umb-2. PLoS One 2008;3:e4069.

33 Wang J, Loberg R, Taichman RS: The pivotal role of cxcl12 (sdf-1)/cxcr4 axis in bone metastasis. Cancer Metastasis Rev 2006;25:573-587.

34 Abe M, Xie W, Regan MM, King IB, Stampfer MJ, Kantoff PW, Oh WK, Chan JM: Single-nucleotide polymorphisms within the antioxidant defence system and associations with aggressive prostate cancer. BJU Int 2011;107:126-134.

35 Yamasaki M, Nomura T, Sato F, Mimata H: Chronic hypoxia induces androgen-independent and invasive behavior in Incap human prostate cancer cells. Urol Oncol 2013;31:1124-1131.

36 Scotton CJ, Wilson JL, Scott K, Stamp G, Wilbanks GD, Fricker S, Bridger G, Balkwill FR: Multiple actions of the chemokine cxcl12 on epithelial tumor cells in human ovarian cancer. Cancer Res 2002;62:5930-5938.

37 Rubin JB, Kung AL, Klein RS, Chan JA, Sun Y, Schmidt K, Kieran MW, Luster AD, Segal RA: A small-molecule antagonist of cxcr4 inhibits intracranial growth of primary brain tumors. Proc Natl Acad Sci USA 2003;100:13513-13518.

38 Zhou Y, Larsen PH, Hao C, Yong VW: Cxcr4 is a major chemokine receptor on glioma cells and mediates their survival. J Biol Chem 2002;277:49481-49487.

39 Salganik RI: The benefits and hazards of antioxidants: Controlling apoptosis and other protective mechanisms in cancer patients and the human population. J Am Coll Nutr 2001;20:464S-472S; discussion 473S-475S.

40 Kukreja P, Abdel-Mageed AB, Mondal D, Liu K, Agrawal KC: Up-regulation of cxcr4 expression in pc-3 cells by stromal-derived factor-1alpha ( $\mathrm{cxcl} 12$ ) increases endothelial adhesion and transendothelial migration: Role of mek/erk signaling pathway-dependent nf-kappab activation. Cancer Res 2005;65:9891-9898.

41 Ngai J, Inngjerdingen M, Berge T, Taskén K: Interplay between the heterotrimeric g-protein subunits galphaq and galphai2 sets the threshold for chemotaxis and tcr activation. BMC Immunol 2009;10:27.

42 Alsayed Y, Ngo H, Runnels J, Leleu X, Singha UK, Pitsillides CM, Spencer JA, Kimlinger T, Ghobrial JM, Jia X, Lu G, Timm M, Kumar A, Côté D, Veilleux I, Hedin KE, Roodman GD, Witzig TE, Kung AL, Hideshima T, Anderson KC, Lin CP, Ghobrial IM: Mechanisms of regulation of cxcr4/sdf-1 (cxcl12)-dependent migration and homing in multiple myeloma. Blood 2007;109:2708-2717.

43 Hwang JH, Chung HK, Kim DW, Hwang ES, Suh JM, Kim H, You KH, Kwon OY, Ro HK, Jo DY, Shong M: Cxc chemokine receptor 4 expression and function in human anaplastic thyroid cancer cells. J Clin Endocrinol Metab 2003;88:408-416. 